\title{
Base Reproduction Number of COVID-19: Statistic Analysis
}

\author{
HongJun Zhu ${ }^{1,2}$, , JiAngPING HuANG ${ }^{1,2}$ AND Xin LiU ${ }^{1,2}$ \\ ${ }^{1}$ School of Software Engineering, Chongqing University of Posts and Telecommunications, Chongqing, 400065, China \\ ${ }^{2}$ Chongqing Engineering Research Center of Software Quality Assurance, Testing and Assessment, Chongqing, 400065, \\ China \\ *zhuhj@ cqupt.edu.cn(HZ)
}

\section{Background}

The coronavirus disease 2019 (COVID-19) has grown up to be a pandemic within a short span of time. The quantification of COVID-19 transmissibility is desired for purposes of assessing the potential for a place to start an outbreak and the extent of transmission in the absence of control measures[1]. It is well known that the transmissibility can be measured by reproduction number. For this reason, the large amount of research focuses on the estimations of reproduction number of COVID-19. However, these previous results are controversial and even misleading. To alleviate this problem, Liu et al[2] advised to use averaging technique. Unfortunately, the fluctuant consequence principally arises from data error or model limitations rather than stochastic noise, where the averaging technique doesn't work well. This has inspired a collection of studies on reproduction number of COVID-19.

\section{Objective}

To find out the reason for estimation change and then the most reliable estimation of base reproduction number.

\section{Definitions}

Reproduction number can be subdivided into basic reproduction number and effective reproductive number. In this paper, our discussion will be confined to basic reproduction number since it is harder to calculate than the other[3]. Even so, the definitions of both are presented here for comparison.

(1) Basic reproduction number (BRN) $R_{0}$ is defined as the expected number of secondary infectious cases generated by an average infectious case in an otherwise uninfected population[1, 4].

(2) Effective reproductive number (ERN), $R_{e}$, is the number of secondary cases generated by an infectious case once an epidemic is underway[1].

BRN $R_{0}$ can be expressed as $R_{0}=k p d$, where $k$ is the number of contacts each infectious individual has per unit time, $p$ is the probability of transmission per contact between an infectious 
medRxiv preprint doi: https://doi.org/10.1101/2020.09.26.20202010; this version posted September 28, 2020. The copyright holder for this preprint (which was not certified by peer review) is the author/funder, who has granted medRxiv a license to display the preprint in perpetuity.

It is made available under a CC-BY-NC-ND 4.0 International license .

case and a susceptible individual, and $d$ is the mean duration of infectiousness[1]. In the absence of control measures, $R_{e}=r R_{0}$, where $r$ is the proportion of the population susceptible [1]. Here, $r<1$.

\section{Data}

Google Scholar and Science Citation Index were used to search for eligible studies about BRN of COVID-19. The estimations of BRN range from 0.3[5] to 8.213[6]. An extensive and resulting description is given in Table 1.

Table 1 . The base reproductive number $R_{0}$

\begin{tabular}{|c|c|c|c|c|c|}
\hline ID & Date & $\boldsymbol{R}_{0}$ & $95 \% \mathrm{CI}$ & Place & First author \\
\hline 1 & $1 / 11$ & 2.2 & $1.4-3.9$ & Wuhan, China & $\operatorname{Li}[4]$ \\
\hline 2 & $1 / 13$ & 0.3 & $0.17-0.44$ & Wuhan, China & $\mathrm{Wu}[5]$ \\
\hline 3 & $1 / 15$ & 2.6 & $2.49-2.63$ & China & Zhao[7] \\
\hline 4 & $1 / 18$ & 2.2 & - & Wuhan, China & Riou[8] \\
\hline 5 & $1 / 18$ & 4.6 & $3.56-5.65(90 \%)$ & Hubei,China & Anastassopoulou[9] \\
\hline 6 & $1 / 22$ & 3.11 & $2.39-4.13$ & Wuhan, China & $\operatorname{Read}[10]$ \\
\hline 7 & $1 / 22$ & 3.15 & - & China & $\operatorname{Tian}[11]$ \\
\hline 8 & $1 / 23$ & 2.9764 & - & Anhui, China & $\operatorname{Tian}[12]$ \\
\hline 9 & $1 / 24$ & 2.24 & $1.96-2.55$ & China & Zhao[13] \\
\hline 10 & $1 / 24$ & 3.58 & $2.89-4.39$ & China & Zhao[13] \\
\hline 11 & $1 / 24$ & 3.2 & $2.7-3.7$ & Wuhan,China & Jung[14] \\
\hline 12 & $1 / 24$ & 2.1 & $2.0-2.2$ & Wuhan,China & Jung[14] \\
\hline 13 & $1 / 28$ & 2.68 & $2.47-2.86$ & Wuhan, China & $\mathrm{Wu}[15]$ \\
\hline 14 & $1 / 30$ & 4.7092 & - & Wuhan, China & Zhao[16] \\
\hline 15 & $1 / 30$ & 5.934 & - & Hubei, China & Zhao[16] \\
\hline 16 & $1 / 30$ & 1.5283 & - & China & Zhao[16] \\
\hline 17 & $2 / 2$ & 2.55 & $2.25-2.96$ & China & Aghaali[17] \\
\hline 18 & $2 / 8$ & 2.79 & - & - & Liu[2] \\
\hline 19 & $2 / 11$ & 5.2 & $5.04-5.47$ & Wuhan, China & Mizumoto[18] \\
\hline 20 & $2 / 29$ & 2.7999 & - & China & Dur-e-Ahmad[19] \\
\hline 21 & $2 / 29$ & 1.78 & $1.36-2.58$ & South Korea & Aghaali[17] \\
\hline 22 & $2 / 29$ & 2.6 & $2.4-2.8$ & Japan & Kuniya[20] \\
\hline 23 & $2 / 29$ & 3.15 & $2.41-3.90$ & - & $\mathrm{He}[21]$ \\
\hline 24 & $3 / 1$ & 2.6 & $2.3-2.9$ & Korea & Zhuang[22] \\
\hline 25 & $3 / 1$ & 3.2 & $2.9-3.5$ & Korea & Zhuang[22] \\
\hline 26 & $3 / 4$ & 2.7 & $2.1-3.4$ & Shahroud, Iran & Khosravi[23] \\
\hline 27 & $3 / 5$ & 2.6 & $2.3-2.9$ & Italy & Zhuang[22] \\
\hline 28 & $3 / 5$ & 3.3 & $3.0-3.6$ & Italy & Zhuang[22] \\
\hline 29 & $3 / 6$ & 2.71 & - & Wuhan, China & Wang[24] \\
\hline 30 & $3 / 7$ & 1.82 & $1.64-2.05$ & Iran & Aghaali[17] \\
\hline 31 & $3 / 7$ & 3.47 & $3.16-3.84$ & Qom, Iran & Aghaali[17] \\
\hline 32 & $3 / 8$ & $2.76-3.25$ & - & Italy & Remuzzi[25] \\
\hline
\end{tabular}


It is made available under a CC-BY-NC-ND 4.0 International license .

\begin{tabular}{rrlcll}
33 & $3 / 9$ & 0.945 & - & Wuhan, China & Ndairoua[26] \\
34 & $3 / 9$ & 3.37 & $3.03-3.81$ & Italy & Aghaali[17] \\
35 & $3 / 10$ & 7.9 & - & Wuhan, China & Zhu[27] \\
36 & $3 / 13$ & $3.2-3.6$ & - & Wuhan, China & Davies[28] \\
37 & $3 / 19$ & 2.3 & - & Ontario, Canada & Tuite[29] \\
38 & $3 / 23$ & 1.593 & $1.582-1.604$ & Korea & Xu[6] \\
39 & $3 / 23$ & 8.213 & $8.139-8.288$ & USA & Xu[6] \\
40 & $3 / 25$ & 5.25 & - & Brazil & Crokidakis[30] \\
41 & $4 / 3$ & 2.58 & - & Costa Rica & Chaves[31] \\
42 & $4 / 5$ & 2.38 & - & Italy & Giordano[32] \\
43 & $4 / 19$ & 5.0692 & - & Heilongjiang, China & Sun[33] \\
44 & $5 / 2$ & 4.86 & - & Iran & Sahafizadeh[34] \\
\hline
\end{tabular}

Note that CI indicates confidence interval. Date means the time when data was collected.

\section{Discussion}

\section{BRN increases with time}

In order to prove the BRN varies with time, the data is divided into two groups: (1) Group I consists of the first 22 estimations of BRN in Table 1; (2) Group II is composed of the last 22 estimations. A statistical hypothesis testing is carried out here. The null hypothesis is that the mean of Group I is no less than Group II. The alternative hypothesis is that the mean of Group II is more than Group I.

Suppose the estimations follows a normal distribution, if the null hypothesis is really true, the test statistic $t$ which follows by the Student's t-distribution should be more than -1.732 with probability $95 \%$, while it is -2.256 according to the data. Therefore, the null hypothesis is rejected and the conclusion is that BRN increases with time.

\section{Difference between methods}

For more than two categories, some of which have small numbers, statistic analysis tends to pool some of the categories together[35]. For this reason, the estimation methods of BRN are divided into three broad categories: (1) methods based on exponent-growth-like models, (2) methods based on SIR epidemic models, and (3) others. For convenience, we will hereafter use 'EX method' to denote the method based on exponent-growth-like models and 'SIR method' to indicate method based on susceptible-infectious-removed (SIR) model.

At first, we suspect there is a significant difference between the groups in variance. This is confirmed by using Levene's test (on the means) since the Levene test statistic 12.28 is far more than 3.23 , which is the upper critical value of the F distribution with 2 and 40 degrees of freedom at a significance level of 0.05 .

For the reason that the variances and sample sizes are unequal across groups, Welch's Test is 
It is made available under a CC-BY-NC-ND 4.0 International license .

used to perform an ANOVA analysis. Here, the null hypothesis is that the means of the three groups are equal. The alternative hypothesis is that more than one group is different from others. From the data, the Welch's test statistic is 3.25 , which more than the upper critical value $F(2$, $14.165)$ at a significance level of 0.1 . As a result, the conclusion can be drawn that more than one group is different from others.

Further, the results of three methods are shown by points in Figure 1. Here, the estimation (3.2-3.6) provided by Davies [28] is substituted by 3.4 for the convenience of pictorial display, and $2.76-3.25$ by 3.005 for the same reason. Regression analysis is implemented separately for each method, as the lines shown in Figure 1. They all show that BRN increases with time. Note that BRN 0.3 is rejected since an epidemic can occur if and only if

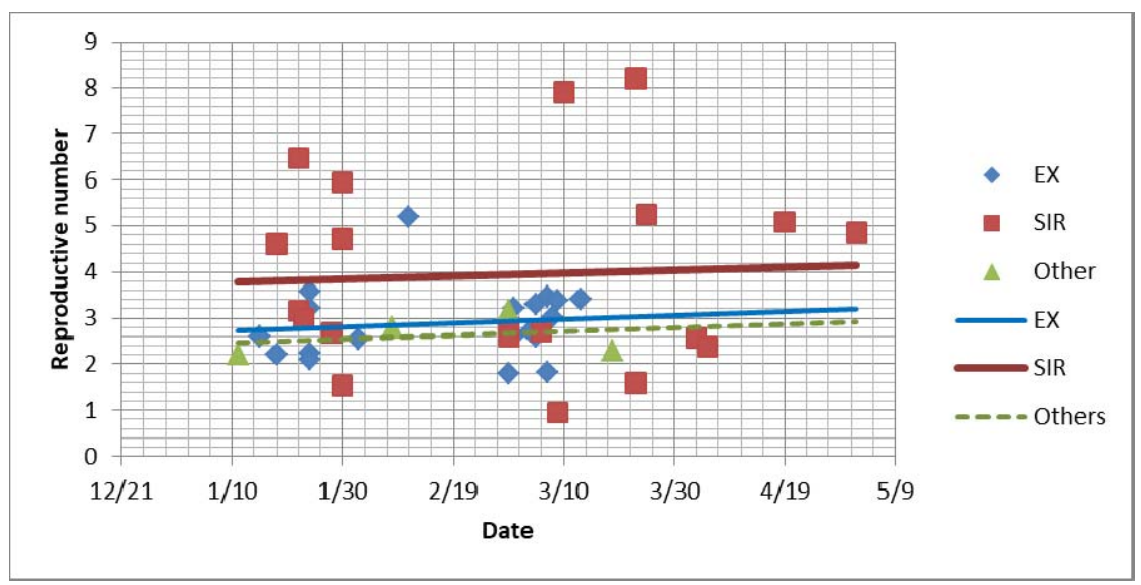

Figure 1. The estimations of BRN based on various methods from the data collected at different time. EX indicates the methods based on exponent-growth-like models and SIR denotes the ones based on SIR models.

The mean and standard deviation of BRN calculated by different classes of method are tabulated in Table 2. It can be observed that the output of SIR methods is higher but more divergent than others. The reason for this is complicated.

Table 2. The mean and standard deviation of BRN calculated by different classes of methods.

\begin{tabular}{lccc}
\hline Parameters & EX method & SIR method & Others \\
\hline Mean & 2.72 & 3.64 & 2.37 \\
Standard deviation & 1.07 & 1.96 & 0.90 \\
\hline
\end{tabular}

At the beginning of the break of COVID-19, the data reported by Wuhan in China is less than the ground truth $[15,36,37]$. Note that EX methods are only competent to calculate BRN from early transmission data[8, 38]. In contrast, SIR methods can be applicable to the whole development process of an epidemic and hence more data can be available. For this reason, the EX methods suffer more ill effects of under-reporting than the SIR methods.

On the other hand, for parameter estimation of epidemic models, the SIR methods usually involve nonlinear optimization, which can easily get stuck into improper local minima[39]. Worse, the parameters are frequently estimated at the cost of model simplification. It is hard to obtain a balance between simplicity and practicality. For this reason, the results of SIR methods are relatively unstable. This may provide an explanation of why the output of SIR methods is more divergent than others. 


\section{Transmissibility increases with time?}

From Figure 1, it is a consensus that the estimations of BRN increase slightly with time. Does the transmissibility of COVID-19 increase as well? In fact, the possible causes of BRN increase are threefold: (1) behavior changes of population, (2) increase of transmissibility of infectious agent and (3) computational errors. Among them, behavioral changes such as wearing mask typically reduce the probability of transmission and ultimately lower rather than enhance BRN. So, if the third cause is impossible either, it is reasonable that the transmissibility of infectious agent of COVID-19 increases with time.

To explore the reason, we carried out a further investigation into the estimation process of BRN. Among them, we found that up to 27 estimates are derived directly from original data reported by Wuhan authority, which is less than the ground truth due to delays in diagnosis and laboratory confirmation in the early stage $[15,36,37]$. Therefore, under-reporting results in an underestimation, which can give a reasonable explanation for BRN increase. As a consequence, no enough evidence demonstrates the transmissibility increase of infectious agent of COVID-19 throughout the world.

\section{Magnitude of BRN}

According to the aforementioned definition, because $k$ and $p$ are related to numerous biological, sociobehavioral, and environmental factors in the special geographical location and historical period, BRN $R_{0}$ is not a biological constant for a pathogen, or a measure of disease severity[3]. Nevertheless, under the same or similar sociobehavioral and environmental conditions, BRN, as an epidemiologic metric, should be higher for the stronger infectious agents. For this reason, we compared the outbreak of COVID-19 with SARS, as tabulated in Table 3. Obviously, the outbreak size of COVID-19 has already far exceeded SARS. In this sense, the BRN of COVID-19 should be far more than that of SARS, i.e., 2.3-3.6[1, 40].

Table 3. The comparison of transmissibility between SARS and COVID-19[41]

\begin{tabular}{|c|c|c|c|c|c|}
\hline item & $\begin{array}{l}\text { Cases in } \\
\text { China }\end{array}$ & $\begin{array}{l}\text { Control measures in } \\
\text { China }\end{array}$ & $\begin{array}{c}\text { Cases in the } \\
\text { world }\end{array}$ & $\begin{array}{l}\text { Countries with } \\
\text { confirmed cases }\end{array}$ & year \\
\hline SARS & 7,747 & No lockdown & 8,422 & 29 & 2002-2003.6 \\
\hline COVID-19 & 89,526 & Lockdown & $20,423,897$ & 212 & 2019-2020.8.13 \\
\hline
\end{tabular}

BRN $R_{0}$ is originally designed to reflect the characteristic of an infectious agent and hence it is required to be measured from the data in the early stage and in the absence of control measures. In this sense, the data of Wuhan, in which COVID-19 began[42], should be the optimal choice to measure the BRN. On the other hand, BRN is more likely to be closer to the estimation from the data of countries with fewer control measures, such as USA. For this reason, here we focus on the BRN of COVID-19 in USA and Wuhan.

The BRN of COVID-19 in USA is estimated to be 8.21 by Xu et al [6]. In fact, after hearing the dangerousness of COVID-19 from Wuhan, American masses may change their behaviors more or less. Therefore, if COVID-19 spreads from USA, the BRN should be more than the estimation 8.21 . 
It is made available under a CC-BY-NC-ND 4.0 International license .

With regard to the BRN of COVID-19 in Wuhan, it is a more complex problem due to under-reporting. Despite a large amount of underestimation appearing in the literature, the several estimations are still similar to the BRN in USA. How to do so? We investigated the data and method, as given in Table 4. It can be observed that most of them utilized compartment models and all of them did not use the raw number of confirmed cases reported by Wuhan authority. Therefore, it is possible to avoid misestimating.

Unlike EX methods, SIR methods can be tested by the predicted results. And, Zhao et al[16] and Zhu et al[27] made a prediction about the end time of COVID-19 in Wuhan, as presented in Table 4. The fact is that, on April 15, the last support medical team was evacuated from Wuhan[43] and Wuhan Thunder Mountain Hospital was officially closed on the same day[44]. For this reason, we believe that the model presented by Zhu et al[27] is more reasonable. In addition, the result computed by Tang[45] demonstrates that the BRN in Wuhan should be more than 6.47, since BRN is typically more than control reproduction number. Given these points, there is strong possibility that the BRN of COVID-19 in Wuhan is 7.9.

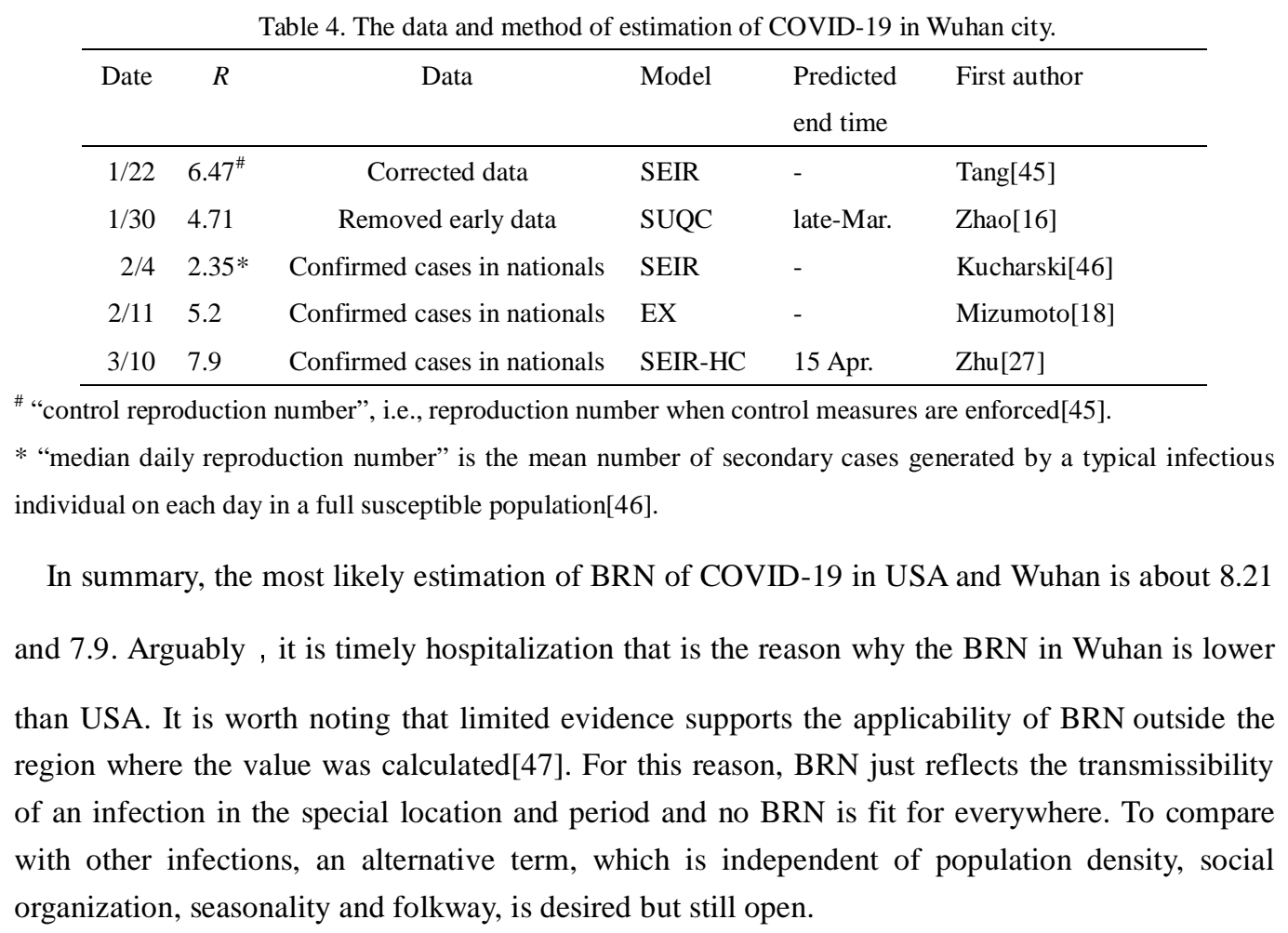

\section{Findings}

The early data is crucial to estimate BRN. Underreporting tends to make an underestimation. In terms of BRN estimation of COVID-19, the SIR-like models are more useful than exponential growth model. The most likely estimation in USA and Wuhan is about 8.21 and 7.9. However, no enough evidence demonstrates the transmissibility increase of infectious agent of COVID-19 throughout the world. Note that it is dangerous to use BRN outside the region where the value was calculated. To compare with other infections, an alternative term, which is independent of population density, social organization, seasonality and folkway, is desired but still open. 
It is made available under a CC-BY-NC-ND 4.0 International license .

\section{Acknowledgment}

This work is supported by National social science foundation of China (16BXW005), Chongqing Basic Science and Frontier Research Project, China (No. cstc2017jcyjAX0007, cstc2017jcyjAX0386), Project Foundation of Chongqing Municipal Education Committee, China (Grant No. 17SKG050).

Reference

1. Marc Lipsitch and et al., Transmission dynamics and control of severe acute respiratory syndrome. Science, 2003. 300(5627): p. 1966-1970.

2. Liu, Y., et al., The reproductive number of COVID-19 is higher compared to SARS coronavirus. Journal of travel medicine, 2020. 27(2): p. 1-4.

3. Delamater, P.L., et al., Complexity of the Basic Reproduction Number $(R(0))$. Emerging infectious diseases, 2019. 25(1): p. 1-4.

4. Li, Q., et al., Early transmission dynamics in Wuhan, China, of novel coronavirus-infected pneumonia. New England Journal of Medicine, 2020. 382(3): p. 1199-1207.

5. Wu, P., et al., Real-time tentative assessment of the epidemiological characteristics of novel coronavirus infections in Wuhan, China, as at 22 January 2020. Euro Surveill, 2020. 25(3): p. 1-7.

6. $\mathrm{Xu}, \mathrm{C}$., et al., Estimation of reproduction numbers of COVID-19 in typical countries and epidemic trends under different prevention and control scenarios. Frontiers of medicine, 2020: p. 1-10.

7. Zhao, S., et al., Estimating the unreported number of Novel Coronavirus (2019-nCoV) cases in China in the first half of January 2020: a data-driven modelling analysis of the early outbreak. Journal of Clinical Medicine, 2020. 9(2): p. 338-343.

8. Riou, J. and C.L. Althaus, Pattern of early human-to-human transmission of Wuhan 2019 Novel Coronavirus (2019-nCoV), December 2019 to January 2020. Euro Surveill, 2020. 25(4): p. 1-5.

9. Anastassopoulou, C., et al., Data-based analysis, modelling and forecasting of the COVID-19 outbreak. PLOS ONE, 2020. 15(3): p. 1-21.

10. Read, J.M., et al., Novel coronavirus 2019-nCoV: early estimation of epidemiological parameters and epidemic predictions. medRxiv, 2020: p. 1-11.

11. Tian, H., et al., An investigation of transmission control measures during the first 50 days of the COVID-19 epidemic in China. Science, 2020. 368: p. 638-642.

12. Tian, J., et al., Modeling analysis of COVID-19 based on morbidity data in Anhui, China. Mathematical Biosciences and Engineering, 2020. 17(4): p. 2842-2852.

13. Zhao, S., et al., Preliminary estimation of the basic reproduction number of novel coronavirus (2019-nCoV) in China, from 2019 to 2020: A data-driven analysis in the early phase of the outbreak. International journal of infectious diseases, 2020. 92: p. 214-217.

14. Jung, S.-M., et al., Real-Time Estimation of the Risk of Death from Novel Coronavirus (COVID-19) Infection: Inference Using Exported Cases. Journal of clinical medicine, 2020. 9(2): p. 523-532.

15. Wu, J.T., K. Leung, and G.M. Leung, Nowcasting and forecasting the potential domestic and international spread of the 2019-nCoV outbreak originating in Wuhan, China: a modeling study. The Lancet, 2020. 395(10225): p. 689-697.

16. Zhao, S. and H. Chen, Modeling the epidemic dynamics and control of COVID-19 outbreak in China. 
Quantitative Biology, 2020. 8(1): p. 11-19.

17. Aghaali, M., et al., Estimation of the serial interval and basic reproduction number of COVID-19 in Qom, Iran, and three other countries: A data-driven analysis in the early phase of the outbreak. Transboundary and Emerging Diseases, 2020: p. 1-9.

18. Mizumoto, K., K. Kagaya, and G. Chowell, Early epidemiological assessment of the transmission potential and virulence of coronavirus disease 2019 (COVID-19) in Wuhan City: China, January-February, 2020. BMC Medicine, 2020: p. 1-9.

19. Dur-e-Ahmad, M. and M. Imran, Transmission Dynamics Model of Coronavirus COVID-19 for the Outbreak in Most Affected Countries of the World. International Journal of Interactive Multimedia and Artificial Intelligence, 2020. 6(2): p. 7-10.

20. Kuniya, T., Prediction of the Epidemic Peak of Coronavirus Disease in Japan. Journal of Clinical Medicine, 2020. 9(3): p. 789-795.

21. He, W., G.Y. Yi, and Y. Zhu, Estimation of the basic reproduction number, average incubation time, asymptomatic infection rate, and case fatality rate for COVID-19: Meta-analysis and sensitivity analysis. Journal of Medical Virology, 2020: p. 1-8.

22. Zhuang, Z., et al., Preliminary estimates of the reproduction number of the coronavirus disease (COVID-19) outbreak in Republic of Korea and Italy by 5 March 2020. International Journal of Infectious Diseases, 2020: p. 308-310.

23. Khosravi, A., et al., The basic reproduction number and prediction of the epidemic size of the novel coronavirus (COVID-19) in Shahroud, Iran. Epidemiology and Infection, 2020. 148: p. 1-7.

24. Wang, L., et al., Modelling and assessing the effects of medical resources on transmission of novel coronavirus (COVID-19) in Wuhan, China. Mathematical Biosciences and Engineering, 2020. 17(4): p. 2936-2949.

25. Remuzzi, A. and G. Remuzzi, COVID-19 and Italy: what next? . The Lancet, 2020. 395: p. 1225-1228.

26. Ndairoua, F., et al., Mathematical modeling of COVID-19 transmission dynamics with a case study of Wuhan. Chaos Solitons \& Fractals, 2020. 135: p. 1-6.

27. Zhu, H., et al., Transmission Dynamics and Control Methodology of COVID-19: a Modeling Study. Applied Mathematical Modelling, 2020.

28. Davies, N.G., et al., Age-dependent effects in the transmission and control of COVID-19 epidemics. Nature medicine, 2020: p. 1-7.

29. Tuite, A.R., D.N. Fisman, and A.L. Greer, Mathematical modelling of COVID-19 transmission and mitigation strategies in the population of Ontario, Canada. Canadian Medical Association Journal, 2020. 192(19): p. 497-505.

30. Crokidakis, N., Modeling the early evolution of the COVID-19 in Brazil: results from a Susceptible-Infectious-Quarantined-Recovered (SIQR) model. International Journal of Modern Physics C, 2020: p. 1-10.

31. Chaves, L.F., et al., COVID-19 basic reproduction number and assessment of initial suppression policies in Costa Rica. Mathematical Modelling of Natural Phenomena, 2020. 15: p. 1-13.

32. Giordano, G., et al., Modelling the COVID-19 epidemic and implementation of population-wide interventions in Italy. Nature Medicine, 2020. 26(6): p. 855-860.

33. Sun, T. and Y. Wang, Modeling COVID-19 epidemic in Heilongjiang province, China. Chaos, Solitons \& Fractals, 2020. 138: p. 1-5.

34. Sahafizadeh, E. and S. Sartoli, Epidemic curve and reproduction number of COVID-19 in Iran. 
medRxiv preprint doi: https://doi.org/10.1101/2020.09.26.20202010; this version posted September 28, 2020. The copyright holder for this preprint (which was not certified by peer review) is the author/funder, who has granted medRxiv a license to display the preprint in perpetuity.

It is made available under a CC-BY-NC-ND 4.0 International license .

Journal of travel medicine, 2020: p. 1-2.

35. McDonald, J.H., Handbook of Biological Statistics. 2009, Baltimore, Maryland, USA: Sparky House Publishing.

36. Nishiura, H., et al., The rate of underascertainment of novel coronavirus (2019-nCoV) infection: estimation using Japanese passengers data on evacuation flights. Journal of Clinical Medicine, 2020. 9(2): p. 419-421.

37. Tuite, A.R. and D.N. Fisman, Reporting, epidemic growth, and reproduction numbers for the 2019 Novel Coronavirus (2019-nCoV) epidemic. Annals of Internal Medicine, 2020. 257(3): p. 1-7.

38. Gerardo, C., et al., Characterizing the reproduction number of epidemics with early subexponential growth dynamics. Jou rnal of the Royal Society Interface, 2016. 13(123): p. 1-12.

39. Zhu, H., et al., Camera calibration from very few images based on soft constraint optimization. Journal of the Franklin Institute-Engineering and Applied Mathematics, 2020. 357(4): p. 2561-2584.

40. Riley, S., et al., Transmission dynamics of the etiological agent of SARS in hong kong: impact of public health interventions. Science, 2003. 300(5627): p. 1961-1966.

41. Park, M., R.S. Thwaites, and P.J.M. Openshaw, COVID-19: Lessons from SARS and MERS. European Journal of Immunology, 2020. 50(3): p. 308-311.

42. Holshue, M.L., et al., First Case of 2019 Novel Coronavirus in the United States. New England Journal of Medicine, 2020. 382(10): p. 929-936.

43. After fighting for 81 days, all the support medical teams were evacuated from Wuhan. 2020 [cited 2020 8.15]; Available from: http://www.wh.gov.cn/sy/whyw/202004/t20200420 1032816.shtml.

44. Yesterday, Wuhan Thunder Mountain Hospital was officially closed. 2020 [cited 2020 8.15]; Available from: http://www.wh.gov.cn/sy/whyw/202004/t20200420 1032816.shtml.

45. Tang, B., et al., Estimation of the transmission risk of the 2019-nCoV and its implication for public health interventions. Journal of Clinical Medicine, 2020. 9(2): p. 462-474.

46. Kucharski, A.J., et al., Early dynamics of transmission and control of COVID-19: a mathematical modelling study. The Lancet Infectious Diseases, 2020. 20: p. 553-558.

47. Ridenhour, B., J.M. Kowalik, and D.K. Shay, Unraveling RO: considerations for public health applications. American journal of public health, 2014. 104(2): p. 32-41. 